\title{
Observations of Energetic-particle Population Enhancements along Intermittent Structures near the Sun from the Parker Solar Probe
}

Riddhi Bandyopadhyay $^{1}$ (D), W. H. Matthaeus ${ }^{1,2}$ (D) T. N. Parashar ${ }^{1,2}$ (D) , R. Chhiber ${ }^{1,3}$ (D), D. Ruffolo ${ }^{4}$ (D), M. L. Goldstein ${ }^{3,5}$ (D), B. A. Maruca ${ }^{1,2}$ (iD) A. Chasapis ${ }^{6}$ (D), R. Qudsi ${ }^{1}$ (i), D. J. McComas ${ }^{7}$ (iD), E. R. Christian ${ }^{3}$ (iD, J. R. Szalay ${ }^{7}$ (D), C. J. Joyce ${ }^{7}$ (D),

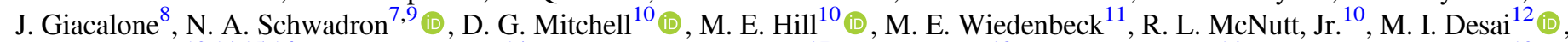
Stuart D. Bale ${ }^{13,14,15,16}$ (D) J. W. Bonnell ${ }^{14}$, Thierry Dudok de Wit ${ }^{17}$, Keith Goetz ${ }^{18}$, Peter R. Harvey ${ }^{14}$, Robert J. MacDowall ${ }^{19}$ (iD), David M. Malaspina $^{20}$ (D), Marc Pulupa ${ }^{14}$ (D), M. Velli ${ }^{21}$ (D), J. C. Kasper $^{22,23}$ (D), K. E. Korreck ${ }^{23}$ (D), M. Stevens ${ }^{23}$ (D), A. W. $\operatorname{Case}^{23}$ (D), and N. Raouafi ${ }^{10}$

${ }^{1}$ Department of Physics and Astronomy, University of Delaware, Newark, DE 19716, USA

${ }^{2}$ Bartol Research Institute, University of Delaware, Newark, DE 19716, USA; whm@udel.edu ${ }^{3}$ NASA Goddard Space Flight Center, Greenbelt, MD 20771, USA

${ }^{4}$ Department of Physics, Faculty of Science, Mahidol University, Bangkok 10400, Thailand

${ }^{5}$ University of Maryland Baltimore County, Baltimore, MD 21250, USA

${ }^{6}$ Laboratory for Atmospheric and Space Physics, University of Colorado Boulder, Boulder, CO 80303, USA

${ }^{7}$ Department of Astrophysical Sciences, Princeton University, Princeton, NJ 08544, USA ${ }^{8}$ University of Arizona, Tucson, AZ 85721, USA

${ }^{9}$ University of New Hampshire, Durham, NH 03824, USA

${ }^{10}$ Johns Hopkins University Applied Physics Laboratory, Laurel, MD 20723, USA

${ }^{11}$ California Institute of Technology, Pasadena, CA 91125, USA

${ }^{12}$ University of Texas at San Antonio, San Antonio, TX 78249, USA

${ }^{13}$ Physics Department, University of California, Berkeley, CA 94720-7300, USA

${ }^{14}$ Space Sciences Laboratory, University of California, Berkeley, CA 94720-7450, USA

${ }_{15}$ The Blackett Laboratory, Imperial College London, London, SW7 2AZ, UK

${ }^{16}$ School of Physics and Astronomy, Queen Mary University of London, London E1 4NS, UK ${ }^{17}$ LPC2E, CNRS and University of Orléans, Orléans, France

${ }^{18}$ School of Physics and Astronomy, University of Minnesota, Minneapolis, MN 55455, USA

${ }^{19}$ Solar System Exploration Division, NASA/Goddard Space Flight Center, Greenbelt, MD 20771, USA

${ }^{20}$ Laboratory for Atmospheric and Space Physics, University of Colorado, Boulder, CO 80303, USA

${ }^{21}$ Department of Earth, Planetary, and Space Sciences, University of California, Los Angeles, CA 90095, USA

${ }^{22}$ Climate and Space Sciences and Engineering, University of Michigan, Ann Arbor, MI 48109, USA

${ }^{23}$ Smithsonian Astrophysical Observatory, Cambridge, MA 02138 USA

Received 2019 September 14; revised 2019 November 29; accepted 2019 December 16; published 2020 February 3

\begin{abstract}
Observations at 1 au have confirmed that enhancements in measured energetic-particle (EP) fluxes are statistically associated with "rough" magnetic fields, i.e., fields with atypically large spatial derivatives or increments, as measured by the Partial Variance of Increments (PVI) method. One way to interpret this observation is as an association of the EPs with trapping or channeling within magnetic flux tubes, possibly near their boundaries. However, it remains unclear whether this association is a transport or local effect; i.e., the particles might have been energized at a distant location, perhaps by shocks or reconnection, or they might experience local energization or re-acceleration. The Parker Solar Probe (PSP), even in its first two orbits, offers a unique opportunity to study this statistical correlation closer to the corona. As a first step, we analyze the separate correlation properties of the EPs measured by the Integrated Science Investigation of the Sun (IS $\odot$ IS) instruments during the first solar encounter. The distribution of time intervals between a specific type of event, i.e., the waiting time, can indicate the nature of the underlying process. We find that the IS $\odot$ IS observations show a power-law distribution of waiting times, indicating a correlated (non-Poisson) distribution. Analysis of low-energy $(\sim 15-200 \mathrm{keV} / \mathrm{nuc})$ IS $\odot$ IS data suggests that the results are consistent with the 1 au studies, although we find hints of some unexpected behavior. A more complete understanding of these statistical distributions will provide valuable insights into the origin and propagation of solar EPs, a picture that should become clear with future PSP orbits.
\end{abstract}

Unified Astronomy Thesaurus concepts: Solar energetic particles (1491); Interplanetary turbulence (830); Interplanetary physics (827); Interplanetary magnetic fields (824); Solar wind (1534); Slow solar wind (1873)

\section{Introduction}

The transport and acceleration of charged energetic particles (EPs) is well known to be intimately related to the properties of plasma turbulence (Jokipii 1966). Transport is particularly sensitive to the magnetic-field structure (Seripienlert et al. 2010; Tooprakai et al. 2016; Malandraki et al. 2019), as well as the distribution of fluctuations over scale (Bieber et al. 1994). Propagation of particles gives rise to a complex relationship of the particle trajectory with the electric fields that ultimately accounts for acceleration in space and astrophysics (Terasawa \& Scholer 1989; Reames 1999; Amato \& Blasi 2018). While many features of EPs can be understood in theoretical frameworks based on quasi-linear theory and random-phase fluctuations, there is an increasing interest in phenomena that can only be understood by taking into account coherent magnetic structures. For us this means the organization of turbulent magnetic fields into flux tubes, and 
their associated coherent structures, such as current sheets, current cores, and secondary flux tubes, plasmoids, and islands that are frequently found on, or near, borders between interacting flux tubes. Moreover, the turbulence cascade can give rise to magnetic "islands" or secondary flux tubes, with dimensions that span a wide range of length scales (Loureiro et al. 2012; Zhdankin et al. 2012, 2013; Wan et al. 2013). Such structures have been suggested to have major effects on charged particle populations, including transport, energization, or both (Khabarova et al. 2015; Khabarova \& Zank 2017). Most of these physical effects and theoretical constructs have found application in the description of solar EPs in the heliosphere, and it is fair to say that many questions remain incompletely settled. One reason is that the different mechanisms and effects cannot always be distinguished at 1 au and beyond, due to the significant ambiguity introduced by the intervening transport and solar wind dynamics.

A main goal of the recently launched Parker Solar Probe (PSP; Fox et al. 2016) has been to disentangle the effects of transport and local acceleration on heliospheric EP populations by making observations that lie much closer to the sources of the EPs than any prior mission. Here, we analyze PSP observations from its first two solar encounters to provide a first look at the statistics of EPs and their relation to magnetic-field roughness or intermittency (Greco et al. 2009a). We employ EP data from the Integrated Science Investigation of the Sun (IS $\odot \mathrm{IS}$ ) instruments (McComas et al. 2016), and magnetic-field data (Bale et al. 2016) from the FIELDS magnetometers on board PSP.

The essential motivation for the present study comes from prior works that found a statistical association of measures of particle energization and measures of magnetic discontinuities using the Partial Variance of Increments (PVIs) method (Greco et al. 2009a, 2018). In particular, Osman et al. (2011) found that the solar wind at $1 \mathrm{au}$ is hotter near high-PVI events (i.e., locally large gradients of the magnetic field). High PVI often corresponds to current structures or current concentrations (Chasapis et al. 2015; Greco et al. 2018). These may be found in the form of sheets, cores, or other formations and are often seen in simulations at boundaries of interacting flux tubes. As such, very strong PVI events have been statistically associated with reconnection events in MHD simulations (Servidio et al. 2011) and in the solar wind (Osman et al. 2014). The PVI method efficiently identifies classical MHD discontinuities, but the method does not distinguish different types of discontinuities such as tangential discontinuities and rotational discontinuities. What we mean by "coherent structure" is simply a concentration of gradients in space. This mathematically requires phase coherence at certain points or regions where the concentration is located. But in principle this idea includes many possible types of structures. Sharp changes in the solar wind magnetic field can be associated with various structures, which may not always be turbulence-associated, e.g., large-scale current sheets, including the heliospheric current sheet, interplanetary shocks, large-discontinuities associated with solar streamers, coronal mass ejections, and corotating interaction region borders, magnetic clouds, and magnetic islands inside a fragmented magnetic cloud. However, since we compute the PVI values using a relatively small-scale increment lag (see Section 4), it is unlikely that the PVI would be sensitive to such large-scale objects. Nevertheless, Tessein et al. (2013, 2015) found a similar, strong statistical association between high-PVI events and enhanced flux of EPs using data from the $A C E$ spacecraft. More recently, Khabarova et al. (2015), Khabarova \& Zank (2017), and Malandraki et al. (2019) found evidence that island- like magnetic structures are associated with higher fluxes of EPs. These observations complement theoretical works (Ambrosiano et al. 1988; Dmitruk et al. 2004; Zank et al. 2014) that describe mechanisms for particle energization by trapping in secondary magnetic structures such as current channels, or small magnetic islands that tend to form during dynamical activity near flux tube boundaries or current sheets. So far, conclusive evidence has not been available to unambiguously distinguish between secondary magnetic structures as transport conduits, a point emphasized by Tessein et al. (2016). Moreover, if secondary islands and flux tubes facilitate and guide transport, there remains the question of where and how the transport originates and what the sources of particles are. For example, is the source close to a nearby CME or interplanetary shock, or is the source distant, perhaps deep in the corona? Although we are not able to answer all these questions in detail, providing new statistical correlations relating SEPs and PVI events during the PSP encounters adds new and important constraints to our understanding of the characteristics of heliospheric EP populations and the mechanisms affecting them. Our analysis also provides a first look at what will eventually be a much more complete survey at even closer distances to the Sun, a goal that will be achieved during subsequent PSP orbits.

\section{PSP Data}

The PSP mission (Fox et al. 2016) completed two orbits between launch on 2018 August 8 and 2019 June 18. The first solar encounter comprised approximately 10 days on each side of the first perihelion at $35.7 R_{\odot}$ on 2018 November 6 , while the second perihelion, also near $35.7 R_{\odot}$, occurred on 2019 April 4. During this passage, the IS $\odot$ IS instrument suite (McComas et al. 2016) performed detailed measurements of solar energetic particles (SEPs; McComas et al. 2019). The FIELDS instrument performed high-cadence measurements of the vector magnetic field (Bale et al. 2019). Ion density and velocity, measured by the Solar Wind Electron Alpha and Protons (SWEAP) instrument suite (Kasper et al. 2016), are used to estimate ion-inertial length and convert spatial lags from temporal lags, using the Taylor hypothesis. We analyze the EP data from the IS $\odot$ IS suite, particularly EPI-Lo ion count rate: total ions from $\sim 15$ to $200 \mathrm{keV} /$ nuc with no mass discrimination, but likely dominated by protons, averaged over all 80 look directions (logical_source: psp_isois-epilo_12-ic, varname: H_CountRate_ChanT). The EPI-Lo instrument measures ions and ion composition from $\sim 20 \mathrm{keV} /$ nucleon to $15 \mathrm{MeV}$ total energy. The magnetic-field data are analyzed at 1 minute cadence (data set: psp-fld-12-mag-RTN-1min). We calculate magnetic-field PVI at 2 minute lag and $4 \mathrm{hr}$ averaging interval, and resample the calculated PVI time series to the EPI-Lo count rate. Figure 1 shows an overview of the first two encounters.

\section{EP Statistics}

Elementary statistical analysis of random signals can reveal a surprising amount of information about the nature of the physical processes that produce the signals. For example, for either discrete or continuous signals, which are functions of time, one may select a threshold value, record the time when the signals exceeds this threshold, wait some time during which the signal falls below this value, and then record the next time at which the signal exceeds the same threshold. This interval, the waiting time, is itself another random variable. The original random variable can be characterized by a probability distribution function such as, for example, a Gaussian distribution if the central limit theorem applies, or a 

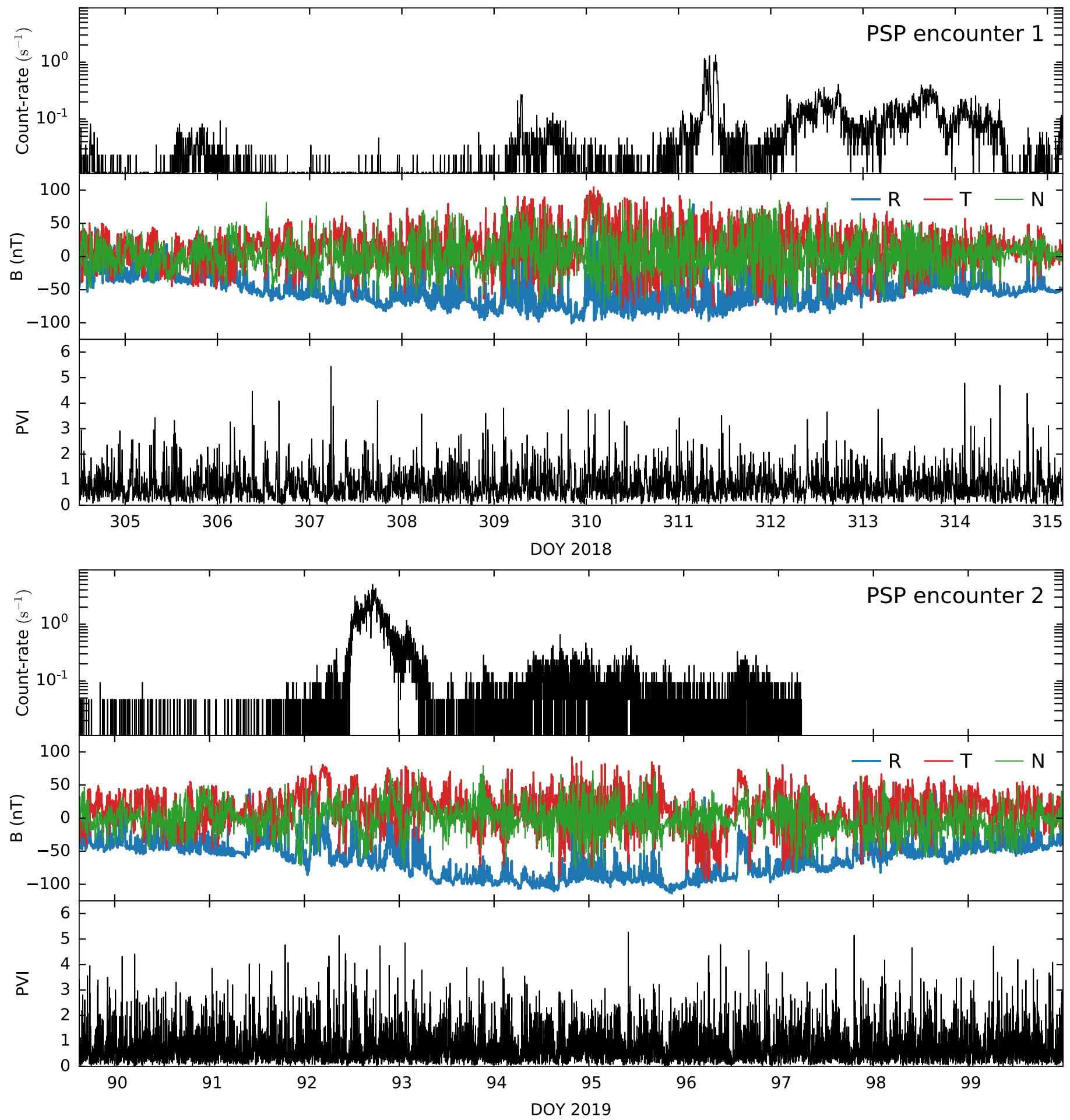

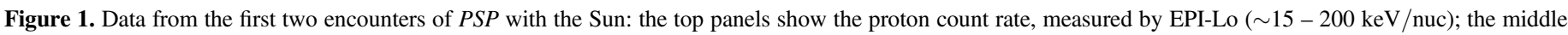
panels plot the RTN components of the magnetic field; and the bottom panels show the magnetic-field PVI.

non-Gaussian distribution with "fat tails" if extreme values have enhanced probability. The distribution of waiting times is a distinct distribution, independent of the distribution of the original variable, and it has independent significance. If the successive waiting times are independent and uncorrelated, the underlying processes is Poissonian, and the distribution of waiting times is expected to be exponential. On the other hand, if the waiting times have a "memory" and are correlated, they will be distributed according to a power law. Examination of waiting times to make this distinction has been a useful tool in the study of processes in geophysics (Lepreti et al. 2001), space physics (Carbone et al. 2006), economics (Greco et al. 2008b), and laboratory materials (Ferjani et al. 2008), to name a few.

We should note that it is not uncommon for signals to be correlated for small time separations (or lengths) up to a certain scale, and for larger scales to become uncorrelated. In this case waiting time distribution would make a transition from a power-law form for smaller separations (up to a correlation 


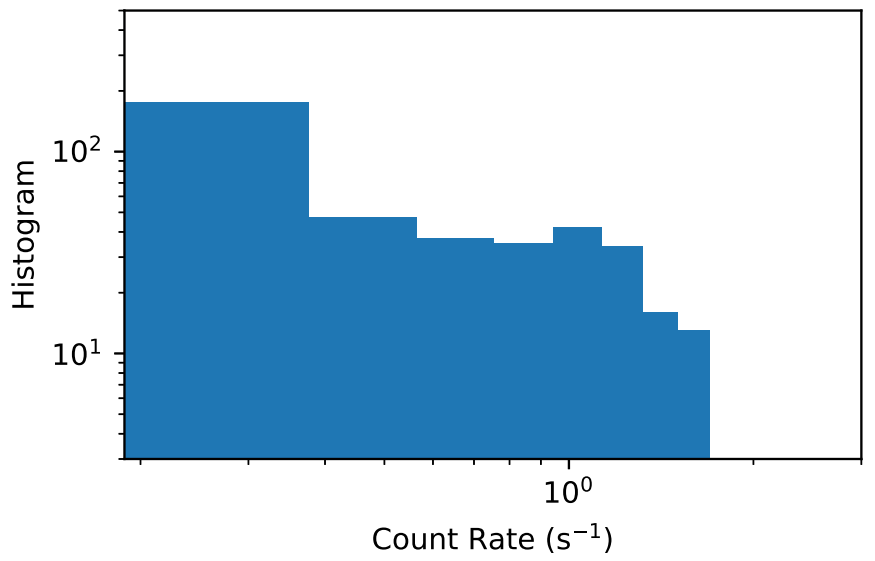

Figure 2. Histograms (showing frequency of occurrence, or number of counts) of count rates measured by IS $\odot$ IS /EPI-Lo for PSP first solar encounter.

scale) to an exponential form. This appears to be the case for magnetic discontinuities in the solar wind measured by the PVI method at $1 \mathrm{au}$ (Greco et al. 2008a). In particular, for waiting times corresponding to spatial scales of about $10^{6} \mathrm{~km}$ or smaller, the waiting times show a clear power-law distribution (Greco et al. 2009a). For larger scales, it becomes exponential and therefore uncorrelated (Greco et al. 2009b).

Here, we will examine waiting time statistics for SEP data measured by the IS $\odot$ IS/EPI-Lo instrument in the first PSP encounter. The purpose is to understand whether in this region, closer to the Sun than previously explored, the occurrence of particle counts is random and uncorrelated, or if the counts are clustered and correlated. Then, in later sections, we examine if apparent clustering is associated with the occurrence of magnetic discontinuities measured using the PVI method. Conclusions regarding these issues are likely to provide information valuable to discovering details of the acceleration and transport mechanisms responsible for observed SEP measurements.

As a first analysis, we examine the distribution of count rates for a particular channel of EPI-Lo data during the first PSP solar encounter, which lasted from 2018 October 31 to 2018 November 12. This is illustrated in Figure 2. One observes that low count rates are much more common than high rates, as expected for SEP data. This episodic property of EP data makes analysis of statistical correlations between SEP counts and any ambient property, such as magnetic field, particularly challenging. In fact for very low count rates, there may be some question as to whether the signal is physically significant, or alternatively, one may be observing a noise signal, due to spurious fluctuations in electronics, for example.

A definitive judgment as to whether particular low-count signals are of significance is difficult or even impossible. However, a reasonable judgment may be made based on the statistics of a particular data record: if the signal exhibits correlations or "clustering," then one might expect that its origin is systematic and likely of physical nature. On the other hand, if the signal is consistent with uncorrelated events or Poisson noise, then one may suspect it is due to random noise or some other memoryless process. In the latter case, it might not contain physical content, or, at least, it demonstrates that the physical processes at work are unrelated. At low count rates Poisson signals would likely indicate the former-a lack of physical content, while at high count rates Poisson noise

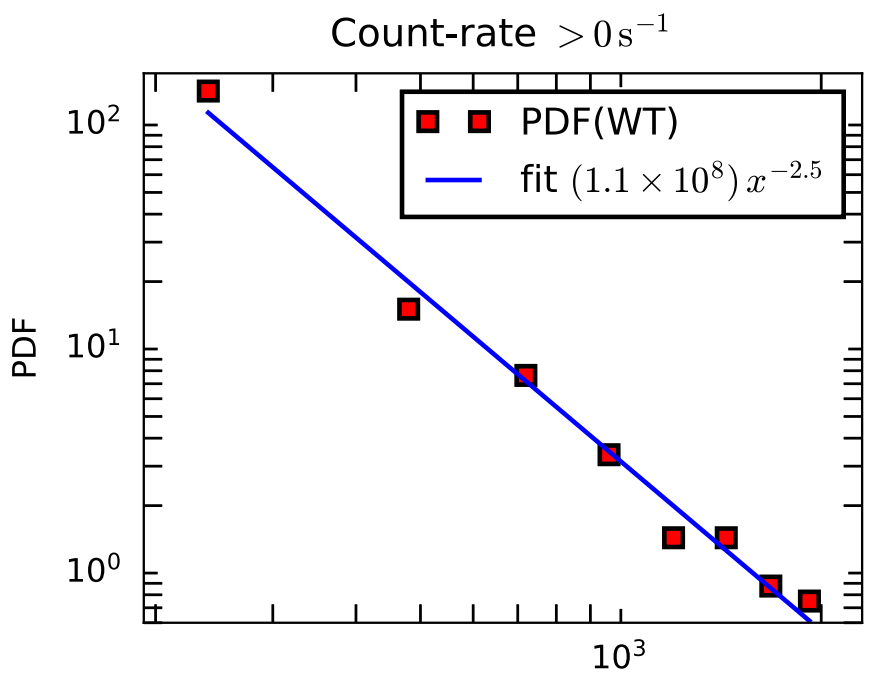

WT (s)

Figure 3. PDF of waiting times between any two nonzero count-rate events for the first encounter. Bins with fewer than five counts are discarded. The powerlaw fit $a x^{b}$ is shown as a solid blue line, where $a=(1.1 \pm 0.9) \times 10^{8}$ and $b=-2.5 \pm 0.1$.

indicates that the physical processes measured are independent. On the other hand, non-Poissonian correlations are very likely to indicate physical correlations. As discussed above, an exponential distribution of waiting times between a signal exceeding a given threshold is associated with a Poisson signal, while a power-law distribution of waiting times suggests physical non-Poissonian correlations (Greco et al. 2008a).

To examine IS $\odot$ IS SEP data for Poissonianity versus clustering, we carry out several related tests of waiting times. First, for all EPI-Lo data in the selected channel during the first encounter, we compute the waiting time distribution between any two detected nonzero count rates. Here, the episodic nature of EP detection is a crucial element. Figure 3 illustrates the result. A power-law fitting is obtained with Pearson's $r$ coefficient (Press et al. 1992) $r^{2}=0.95$. At the same time, an exponential fitting (not reported here) results in worse quality of fitting with $r^{2}=0.41$. It is apparent that the waiting time distribution is consistent with a power law, indicating clustering.

As a next step, we examine waiting times of IS $\odot$ IS data by selecting the signals based on a threshold value of the count rate. For low count-rate signals, we record the waiting times between signals, which are less than $0.02 \mathrm{~s}^{-1}$. For high countrate signals, we select the signals with count rates $>0.1 \mathrm{~s}^{-1}$. For both low and high count-rate signals, these conditional waiting time distributions are shown in Figure 4. For low count rates, $<0.02 \mathrm{~s}^{-1}$, we see in the left panel that the waiting time distribution is clearly exponential, supporting the conclusion that one-event level data is Poisson noise. The Pearson's $r$ coefficient is $r^{2}=0.99$ for exponential fit and $r^{2}=0.71$ for a power-law fit (not shown here), implying better agreement with the exponential fitting shown here. However, if the waiting times are computed for higher count rates $>0.1 \mathrm{~s}^{-1}$ only, a power-law distribution is recovered. Here, although the number of points to fit is low (4), the power-law fit yields a value of $r^{2}=0.97$, while an exponential fit (not shown) performs worse 

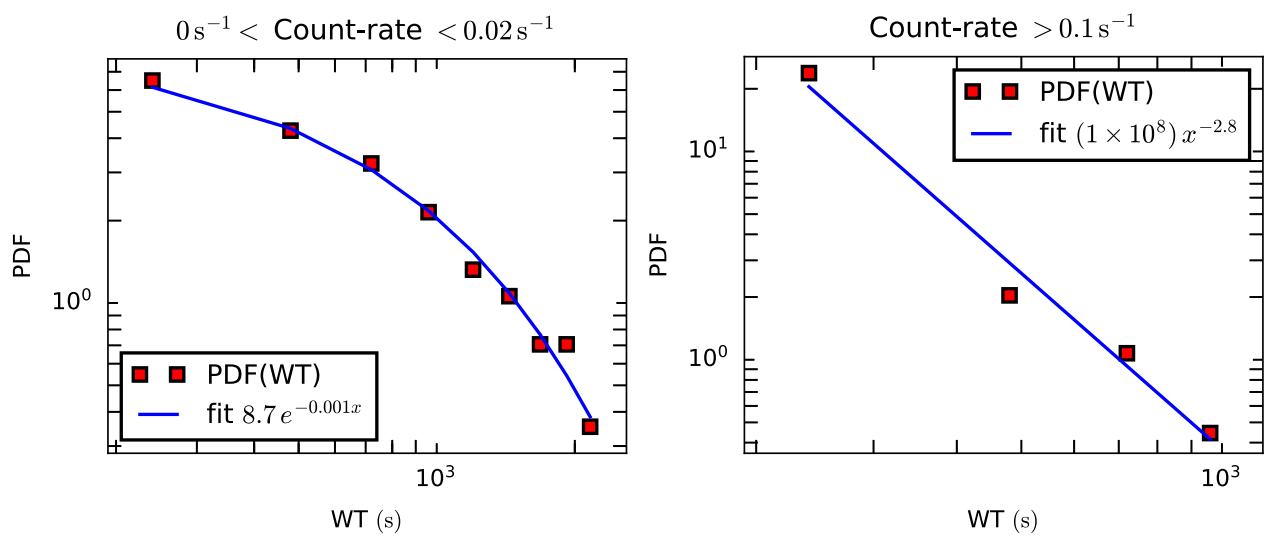

Figure 4. PDFs of waiting times between events with (left) $0 \mathrm{~s}^{-1}<$ count rate $<0.02 \mathrm{~s}^{-1}$ and (right) count rate $>0.1 \mathrm{~s}^{-1}$, for the first $P S P$ encounter. The exponential $c e^{-x d}$ and the power law $a x^{b}$ are shown as solid blue lines. The parameters and uncertainties of the fit are (for the left panel) $c=8.7 \pm 0.8$ and $d=(-144 \pm 7) \times 10^{-5}$; (for the right panel) $a=(1 \pm 1) \times 10^{8}$, and $b=-2.8 \pm 0.3$. Bins with fewer than five counts have been discarded.

with $r^{2}=0.76$. Similar results are obtained for the second solar encounter as well (not shown here).

\section{EPs and Coherent Structures}

A main challenge of statistical analysis of suprathermalparticle data is that these particles are already very rare. Therefore, usually long durations of data collection are required to deduce any statistical trend in the investigations. The first two solar encounters by PSP produce reasonably well-populated SEP measurements to carry out some statistical correlations.

A turbulent system naturally generates patchy or intermittent fluctuations and concentrations of gradients of the primitive variables (Sreenivasan \& Antonia 1997; Matthaeus et al. 2015). A practical technique for identifying these coherent structures is the method of PVI (Greco et al. 2008a, 2009a, 2018). This technique uses magnetic-field data to identify small-scale structures such as current concentration. For single-spacecraft measurements, this method involves calculation of temporal increments of the magnetic field $\left|\Delta \boldsymbol{B}_{\tau}(t)\right|=|\boldsymbol{B}(t+\tau)-\boldsymbol{B}(t)|$. From that, the normalized PVI index at a lag of $\tau$ is given by

$$
\operatorname{PVI}(t)=\sqrt{\frac{\left|\Delta \boldsymbol{B}_{\tau}(t)\right|^{2}}{\left\langle\left|\Delta \boldsymbol{B}_{\tau}(t)\right|^{2}\right\rangle}},
$$

where $\langle\cdots\rangle$ denotes a time average over a suitably large trailing sample, computed along the time series. Using the Taylor hypothesis (Taylor 1938), then, one can convert temporal scales to length scales. At a heuristic level, one may think of PVI as a measurement of the roughness of the magnetic field. Roughness will be greater in regions where the fluctuations are larger, but PVI is most sensitive to relative roughness, in comparison to the regional values. The increment of a turbulent field has long been of central importance in turbulence research, with particular importance having been given to moments of the increment, the so-called structure functions (Monin \& Yaglom 1971, 1975). The square of PVI, as defined in Equation (1), is related to the secondorder structure function, but PVI is distinct in that it is a pointwise, rather than an averaged quantity. The PVI method is one among several that have been developed for identifying discontinuities in turbulent flows, such as the Tsurutani-Smith method (Tsurutani \& Smith 1979), wavelet-based Local-Intermittency Measure (Veltri \& Mangeney 1999; Farge et al. 2001), and Phase Coherence Index (Hada et al. 2003). Greco et al. (2018) discuss a

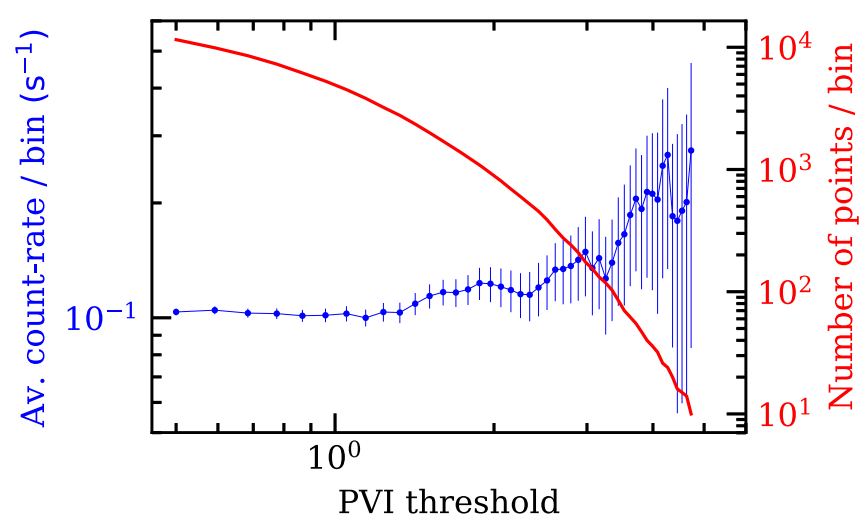

Figure 5. Average energetic-particle count rate plotted against PVI threshold for the first two solar encounters. A statistically significant increase in average count rates at higher PVI is noted.

comparison of some of these methods with the PVI technique. We use the PVI method here for its simplicity and its direct relationship to increment statistics. Here, we are interested in fluctuations near the inertial range of scales associated with the turbulence power spectrum (Kolmogorov 1941; Matthaeus \& Goldstein 1982). The dynamics at these scales are governed by local-in-scale, non-linear processes and kinetic effects do not become dominant here. In this work, we calculate the PVI values for 2 minute $\left(\sim 42,000 \mathrm{~km} \sim 2800 d_{\mathrm{i}}\right)$ lag, which is well within the inertial range, for the first two encounters. The averaging interval is $4 \mathrm{hr}$. Here, $d_{\mathrm{i}}$ is the ion-inertial length, defined as $d_{\mathrm{i}}=c / \omega_{\mathrm{pi}}=\sqrt{m_{\mathrm{i}} \epsilon_{0} c^{2} / n_{\mathrm{i}} e^{2}}$, where $c$ is the speed of light in vacuum, $\omega_{\mathrm{pi}}$ is the proton plasma frequency, $m_{\mathrm{i}}$ is the proton mass, $\epsilon_{0}$ is the vacuum permittivity, $n_{\mathrm{i}}$ is the number density of protons, and $e$ is the proton charge.

To study the association of EP measurements with magneticfield structures, we calculate the average count rate for a given PVI threshold value. The blue dots in Figure 5 plot the average EP flux per PVI bin against PVI. Error bars are also shown as vertical lines. The uncertainty is estimated as $\sigma_{i} / \sqrt{m}$, where $\sigma_{i}$ is the standard deviation of the points in the $i$ th bin and $m$ is the number of points in that bin. The number of samples (right axis) for each PVI bin is shown for all data as a red solid line. For PVI greater than 1 a rough, positive correlation with the count rate can be observed, with moderate statistical significance. Although, the error bars become large at higher PVI 

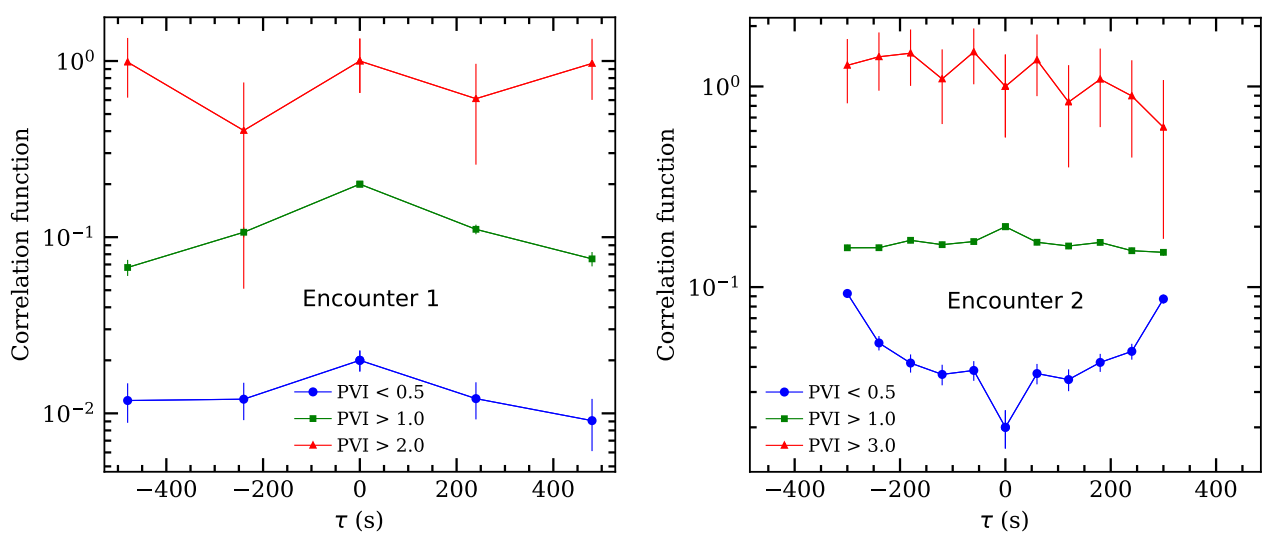

Figure 6. Time-lagged correlation of average energetic-particle count rate and PVI for different PVI threshold values for the first two encounters (left: encounter 1; right: encounter 2). The correlation functions have been shifted in the vertical direction for clarity. Note that the measurement cadences were 4 minutes and 1 minute during the first and second encounter passes, respectively.

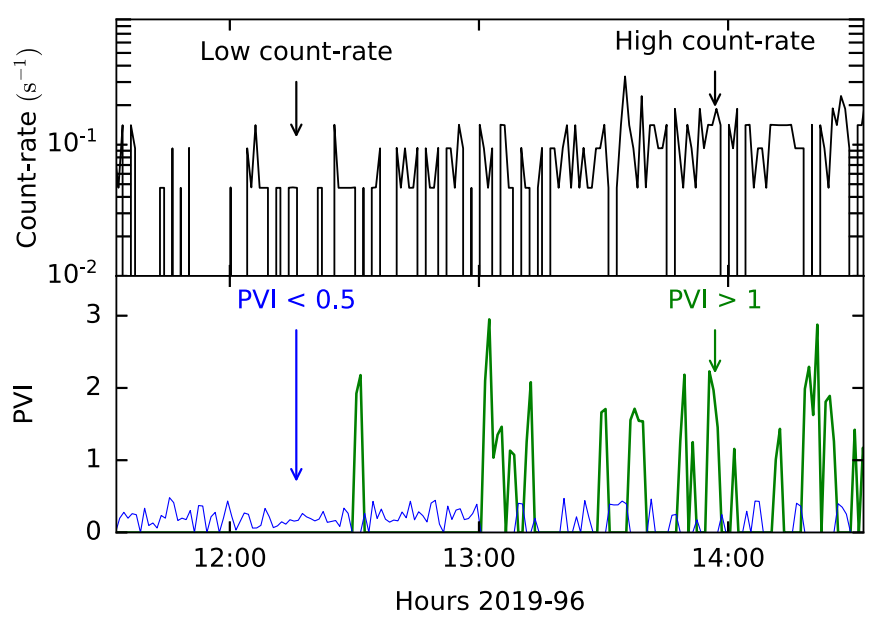

Figure 7. Example of observations of low (or high) energetic-particle count rates occurring near low (or high) PVI events, from encounter 2. The $\mathrm{PVI}<0.5$ values are shown as a thin blue line, and the PVI $>1$ values are shown as a thick green line.

values ( $>3$ ), on average EP count rates and PVI threshold appear to be qualitatively correlated. This indicates that there is a higher probability of finding high EP count rates near intense coherent structures. A similar result was found at 1 au using $A C E$ data (Tessein et al. 2016).

To further test the proximity of EPs with rough magneticfield structures, we calculate a time-lagged cross-correlation between the two quantities. The normalized cross-correlation between two quantities, e.g., the EP count rate $(C(t))$ and magnetic-field PVI, is defined as $R(\tau) / R(0)$, where,

$$
R(\tau)=\langle C(t) \operatorname{PVI}(t+\tau)+C(t+\tau) \operatorname{PVI}(t)\rangle .
$$

Here $\langle\cdots\rangle$ denotes the time average over the time series. Figure 6 shows this cross-correlation computed from subsets of the IS $\odot$ IS data during the first two encounters for several PVI thresholds. The left panel shows the lagged correlation for the first encounter, and the right panel shows the same quantity computed from the second encounter data. The different curves have been vertically shifted from the original value of unity at zero lag, for ease of visualization. The error bars are shown as vertical lines, when they are bigger than the plotting symbols. We only plot those points for which the relative errors are smaller than unity.

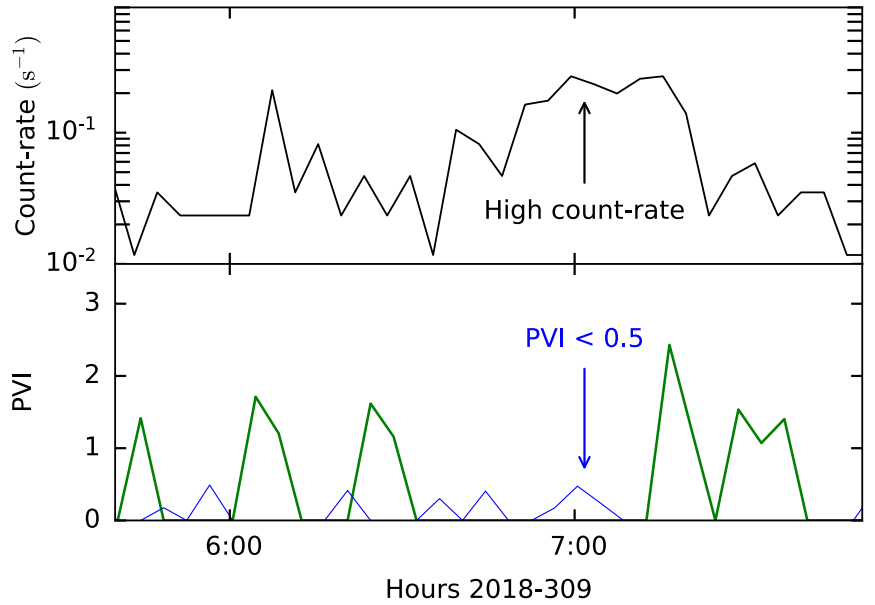

Figure 8. Example of observations of high energetic-particle count rates occurring near low-PVI events, from encounter 1. Plotting conventions are the same as those of Figure 7.

For the first two encounters, the correlation times are close to $\tau_{\text {corr }} \sim 500 \mathrm{~s}$ (Chhiber et al. 2020). To avoid sampling the large-scale inhomogeneities, we calculate the cross-correlations for only a fraction of the average correlation time $\tau<500 \mathrm{~s}$.

For the cases with PVI $>1$, for both encounters, correlation peaks near zero lag, meaning that two quantities are correlated and changing together in time. However, an interesting trend can be observed from the PVI $<0.5$ curve for encounter 2 . In this case, the correlation is slightly suppressed near zero lag and then increases far from the midpoint, in the direction of negative lags (earlier times). This indicates that very low SEP counts are found in regions of very smooth magnetic fields. Possible explanations for these correlations are discussed briefly in the discussion section. A sample case for this type of correlation between energetic-particle data and PVI level is shown in Figure 7. The interval from encounter 2 shows that in the first part of the interval, when the count rates are somewhat low, the PVI values are also small, on average. In the later part, both count rate and PVI values are high, most of the time.

Conversely, in regions distant from a known smooth magnetic-field location, one finds higher EP counts. On the other hand, the correlation for very low PVI in encounter 1 actually peaks at zero lag. This implies that an enhanced EP count rate may be found near even relatively weak magneticfield gradients. Figure 8 exhibits an example of an event when 


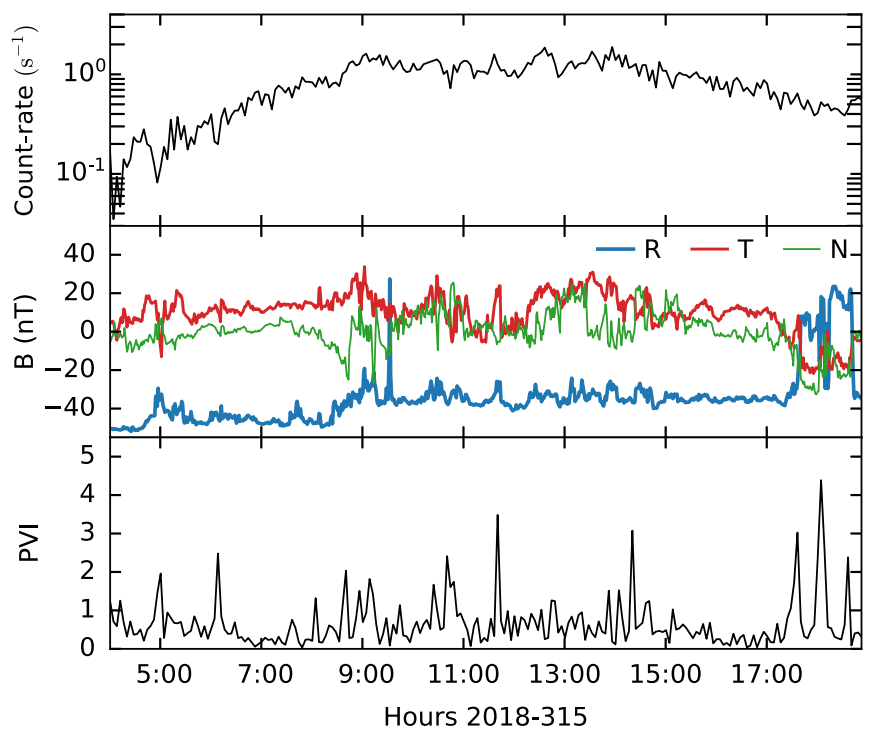

Figure 9. Time-series plot of (top) EPI-Lo proton count rates, (middle) the magnetic-field components, and (bottom) the magnetic-field PVI.

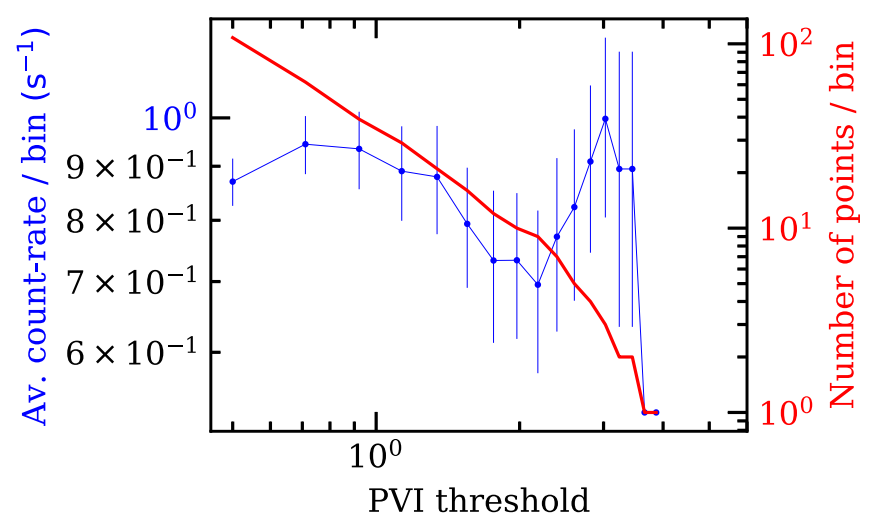

Figure 10. Average energetic-particle count rate plotted against PVI threshold for the dispersive event on 2018-315.

the count rate is high but the PVI is smaller than 0.5. One may note, however, that in this event the region of higher particle counts is approximately bounded on each side by elevated PVI (although still relatively low values). This is reminiscent of the suggestion made by Tessein et al. (2016) that PVI events at edges of particle enhancements may sometimes be a signature of confinement (see also Seripienlert et al. 2010).

Another contrasting feature from Figure 6 is that for encounter 2, the PVI $>3$ curve is somewhat skewed, with more average EPs before the PVI events (negative time lag) and fewer counts after (positive time lag). This trend can be qualitatively interpreted in the following way: let us assume that, except for shocks, sharp magnetic discontinuities (high PVI) lie near the flux tube boundaries. Close to the Sun, where these structures are being accelerated rapidly, there may be a pile-up of EPs just at the front edge of the boundaries, while the density of EPs inside the flux tube is low, comparatively. This is an intriguing possibility, though at present the results are qualitative, so a firm conclusion cannot be reached at this stage.

These interesting features are only prominent for the second encounter analysis, presumably due to larger sample size. The first encounter results show peaked correlation at zero lag for all three PVI threshold values that are shown. Nevertheless, the rate of the decrease from zero lag, along both directions, becomes steeper for higher values of PVI threshold. We refrain from interpreting any deep conclusions from these results at this stage.

\section{A Dispersive Event}

A dispersive event, thought to be associated with a weak CME, was observed near the end of the first encounter on 2018-315 from 04:00 to 19:00 UTC, approximately (see McComas et al. 2019; Giacalone et al. 2020; also see K. E. Korreck et al. 2019, in preparation; Nieves-Chinchilla et al. 2020; Rouillard et al. 2020). Figure 9 plots the time series of the relevant quantities for this time interval. In this section, we repeat the analyses in the previous section for this particular event separately. Statistical correlations derived from data when the source is known to be relatively nearby, such as this event, present an interesting point of contrast to the general statistics derived from the entire encounter presented in the previous section.

Figure 10 employs the data from the dispersive event, and plots the conditionally averaged EP count rate for various selected PVI thresholds, similar to Figure 5. Even though the calculations now involve only this limited data interval, a positive correlation between average EP populations and coherent structures is again observed. While this result appears to be statistically significant, the regional (time-lagged) correlation results, derived from the dispersive event, are rather weak and might be inconclusive; therefore, they are discussed in a brief Appendix.

\section{Discussion and Conclusions}

In this paper, by performing a study of joint statistics of IS $\odot$ IS /EPI-Lo samples, in conjunction with FIELDS/MAG measurements, we have presented first results for the association of EPs and magnetic-field structures in the inner heliosphere, most likely close to the Alfvén surface.

In a first step, we used a waiting time analysis for different count-rate thresholds, and reached a conclusion about the clustering or randomness of the samples. Specifically, we find that all but the lowest count rate IS $\odot$ IS data exhibit waiting times consistent with clustering, supporting the interpretation that physical correlations are detected.

Several important extensions to this simple exercise remain to be performed. Instead of categorizing the data by simply count rate, one may undertake a more advanced survey, based on simultaneous EPI-Lo and EPI-Hi measurements. Due to several external factors, e.g., light contamination, temperature, cosmic rays, etc., some weak count rates may be based on real activity, and may not just be due to background noise. A waiting time study, similar to the one presented here, might be able to differentiate these kind of signals.

Another task might be to compare the results for different energy ranges of the instrument. It is expected that the method would be able to distinguish between counts in the very high energy range $(>1 \mathrm{MeV})$, which are presumably dominated by background noise, and below $200 \mathrm{keV}$, which are expected to have foreground contamination.

The second stage of the analysis examined the conditionally averaged EP count rates, based on a simplistic measure of 
the local intermittency, or roughness of the magnetic field. The method employed a PVI analysis, with the purpose of selecting structures with strong magnetic gradients, indicating the possible presence of coherent structures. The results for the first two PSP encounters appear to be consistent with the conclusion that solar EPs are likely correlated with coherent magnetic structures. This result suggests the possibility that EPs might be concentrated near magnetic flux tube boundaries, although other interpretations are also possible (see, e.g., Kittinaradorn et al. 2009; Seripienlert et al. 2010; Tooprakai et al. 2016; Malandraki et al. 2019). This effect has also been reported at 1 au and may be associated either with transport effects, or even local acceleration processes (Tessein et al. 2016; Khabarova et al. 2015). An analysis focusing on a single day during which a dispersive SEP event occurred suggests similar conclusions. However, to draw firmer conclusions we must await data from future PSP orbits. Other possible explanations may emerge. For example, this inverse correlation for PVI $<0.5$ might be an indication that pressure induced locally by EPs smooths out the field. The fact that this occurs prominently in the second solar encounter may be an indication of increased solar activity during this encounter. Another possible interpretation is that the low-PVI regions have lowbeta structures, so the EP counts are weak in these regions. These ideas require further testing from SWEAP data (Kasper et al. 2019), when available in the future. As further data from these type of events are accumulated by PSP and future missions like Solar Orbiter (Müller et al. 2013), and as regions even closer to the corona are explored, it is likely that the crucial questions regarding the relative role of transport effects and sources of energization will be further clarified.

Parker Solar Probe was designed, built, and is now operated by the Johns Hopkins Applied Physics Laboratory as part of NASA's Living with a Star (LWS) program (contract NNN06AA01C). Support from the LWS management and technical team has played a critical role in the success of the Parker Solar Probe mission. We are deeply indebted to everyone who helped make the PSP mission possible. In particular, we thank all of the outstanding scientists, engineers, technicians, and administrative support people across all of the IS $\odot$ IS institutions that produced and supported the IS $\odot$ IS instrument suite and support its operations and the scientific analysis of its data. We also thank the FIELDS and SWEAP teams for cooperation. The IS $\odot$ IS data and visualization tools are available to the community at: https://spacephysics. princeton.edu/missions-instruments/isois; data are also available via the NASA Space Physics Data Facility (https://spdf. gsfc.nasa.gov/). This research was partially supported by the Parker Solar Probe Plus project through Princeton/IS $\odot$ IS subcontract SUB0000165, and in part by NSF-SHINE AGS1460130, NASA Heliospheric Guest Investigator grant No. 80NSSC19K0284, and grant RTA6280002 from Thailand Science Research and Innovation. S.D.B. acknowledges the support of the Leverhulme Trust Visiting Professorship program.

\section{Appendix}

Here, we provide a calculation similar to Figure 6, but only for the dispersive event on 2018-315. The results in Figure 11 are similar to those in Figure 6, computed from longer data sets, encompassing the first two encounters. However, due to

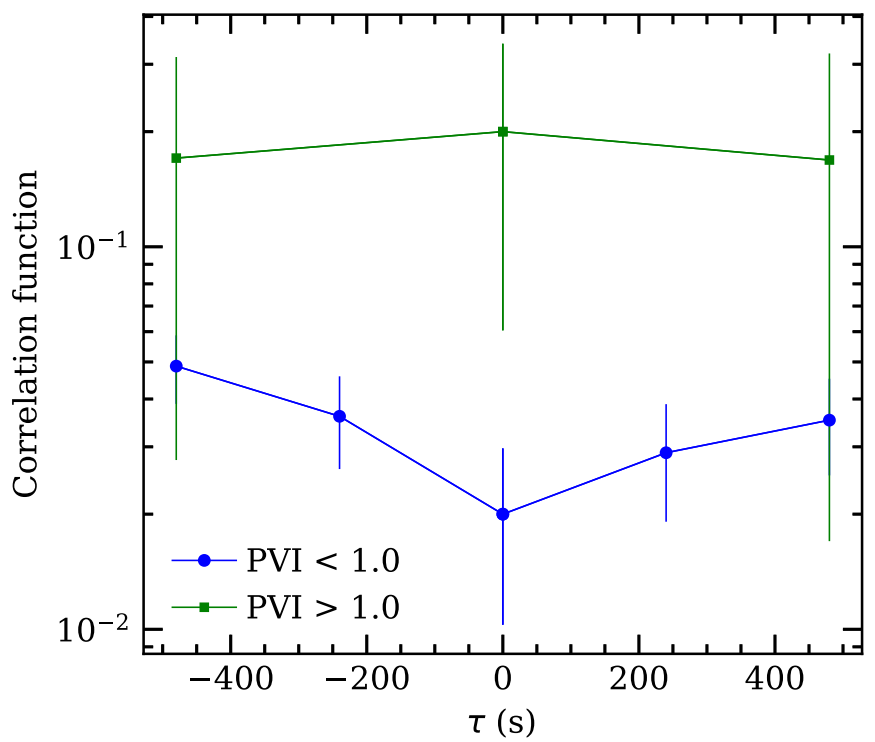

Figure 11. Time-lagged correlation of average energetic-particle count rate and PVI for different PVI threshold values for the dispersive event on 2018-315.

low sample size, the prominence is weak and the conclusions are qualitative, at best.

\section{ORCID iDs}

Riddhi Bandyopadhyay (ib https://orcid.org/0000-00026962-0959

W. H. Matthaeus (1) https://orcid.org/0000-0001-7224-6024

T. N. Parashar (i) https://orcid.org/0000-0003-0602-8381

R. Chhiber (i) https://orcid.org/0000-0002-7174-6948

D. Ruffolo (i) https://orcid.org/0000-0003-3414-9666

M. L. Goldstein (1) https://orcid.org/0000-0002-5317-988X

B. A. Maruca (i) https://orcid.org/0000-0002-2229-5618

A. Chasapis (1) https://orcid.org/0000-0001-8478-5797

R. Qudsi (1) https://orcid.org/0000-0001-8358-0482

D. J. McComas (i) https://orcid.org/0000-0001-6160-1158

E. R. Christian (ํ) https://orcid.org/0000-0003-2134-3937

J. R. Szalay (i) https://orcid.org/0000-0003-2685-9801

C. J. Joyce (1) https://orcid.org/0000-0002-3841-5020

N. A. Schwadron (1) https://orcid.org/0000-0002-3737-9283

D. G. Mitchell 1 https://orcid.org/0000-0003-1960-2119

M. E. Hill ๑i https://orcid.org/0000-0002-5674-4936

M. I. Desai (1) https://orcid.org/0000-0002-7318-6008

Stuart D. Bale (1) https://orcid.org/0000-0002-1989-3596

Robert J. MacDowall (1) https://orcid.org/0000-0003-

3112-4201

David M. Malaspina @ https://orcid.org/0000-0003-

1191-1558

Marc Pulupa (ib https://orcid.org/0000-0002-1573-7457

M. Velli (i) https://orcid.org/0000-0002-2381-3106

J. C. Kasper (1) https://orcid.org/0000-0002-7077-930X

K. E. Korreck (1) https://orcid.org/0000-0001-6095-2490

M. Stevens 1 i https://orcid.org/0000-0002-7728-0085

A. W. Case (1) https://orcid.org/0000-0002-3520-4041

\section{References}

Amato, E., \& Blasi, P. 2018, AdSpR, 62, 2731

Ambrosiano, J., Matthaeus, W. H., Goldstein, M. L., \& Plante, D. 1988, JGR, 93, 14383

Bale, S. D., Goetz, K., Harvey, P. R., et al. 2016, SSRv, 204, 49 
Bale, S. P., Badman, S. T., Bonnell, J. W., et al. 2019, Natur, 576, 237

Bieber, J. W., Matthaeus, W. H., Smith, C. W., et al. 1994, ApJ, 420, 294

Carbone, V., Sorriso-Valvo, L., Vecchio, A., et al. 2006, PhRvL, 96, 128501

Chasapis, A., Retino, A., Sahraoui, F., et al. 2015, ApJL, 804, L1

Chhiber, C., Goldstein, M. L., Maruca, B. A., et al. 2020, ApJS, doi:10.3847/ $1538-4365 / \mathrm{ab} 53 \mathrm{~d} 2$

Dmitruk, P., Matthaeus, W. H., \& Lanzerotti, L. J. 2004, GeoRL, 31, 21805

Farge, M., Pellegrino, G., \& Schneider, K. 2001, PhRvL, 87, 054501

Ferjani, S., Sorriso-Valvo, L., De Luca, A., et al. 2008, PhRvE, 78, 011707

Fox, N. J., Velli, M. C., Bale, S. D., et al. 2016, SSRv, 204, 7

Giacalone, J., Mitchell, D. G., Allen, R. C., et al. 2020, ApJS, doi:10.3847/ $1538-4365 / \mathrm{ab} 5221$

Greco, A., Chuychai, P., Matthaeus, W. H., Servidio, S., \& Dmitruk, P. 2008a, GeoRL, 35, L19111

Greco, A., Matthaeus, W. H., Perri, S., et al. 2018, SSRv, 214, 1

Greco, A., Matthaeus, W. H., Servidio, S., Chuychai, P., \& Dmitruk, P. 2009a, ApJL, 691, L111

Greco, A., Matthaeus, W. H., Servidio, S., \& Dmitruk, P. 2009b, PhRvE, 80, 046401

Greco, A., Sorriso-Valvo, L., Carbone, V., \& Cidone, S. 2008b, PhyA, 387,4272

Hada, T., Koga, D., \& Yamamoto, E. 2003, SSRv, 107, 463

Jokipii, J. R. 1966, ApJ, 146, 480

Kasper, J. C., Abiad, R., Austin, G., et al. 2016, SSRv, 204, 131

Kasper, J. C., Bale, S. D., Belcher, J. W., et al. 2019, Natur, 576, 228

Khabarova, O., Zank, G. P., Li, G., et al. 2015, ApJ, 808, 181

Khabarova, O. V., \& Zank, G. P. 2017, ApJ, 843, 4

Kittinaradorn, R., Ruffolo, D., \& Matthaeus, W. H. 2009, ApJL, 702, L138

Kolmogorov, A. N. 1941, DoSSR, 32, 16

Lepreti, F., Carbone, V., \& Veltri, P. 2001, ApJL, 555, L133

Loureiro, N. F., Samtaney, R., Schekochihin, A. A., \& Uzdensky, D. A. 2012, PhPl, 19, 042303

Malandraki, O., Khabarova, O., Bruno, R., et al. 2019, ApJ, 881, 116

Matthaeus, W. H., \& Goldstein, M. L. 1982, JGR, 87, 6011

Matthaeus, W. H., Wan, M., Servidio, S., et al. 2015, RSPTA, 373, 20140154

McComas, D. J., Alexander, N., Angold, N., et al. 2016, SSRv, 204, 187

McComas, D. J., Christian, E. R., Cohen, C. M. S., et al. 2019, Natur, 576, 223

Monin, A. S., \& Yaglom, A. M. 1971, Statistical Fluid Mechanics, Vol. 1 (Cambridge, MA: MIT Press)
Monin, A. S., \& Yaglom, A. M. 1975, Statistical Fluid Mechanics, Vol. 2 (Cambridge, MA: MIT Press)

Müller, D., Marsden, R. G., St. Cyr, O. C., \& Gilbert, H. R. 2013, SoPh, 285,25

Nieves-Chinchilla, T., Szabo, A., Korreck, K. E., et al. 2020, ApJS, doi:10. 3847/1538-4365/ab61f5

Osman, K. T., Matthaeus, W. H., Gosling, J. T., et al. 2014, PhRvL, 112 , 215002

Osman, K. T., Matthaeus, W. H., Greco, A., \& Servidio, S. 2011, ApJL, 727, L11

Press, W. H., Teukolsky, S. A., Vetterling, W. T., \& Flannery, B. P. 1992, Numerical Recipes The Art of Scientific Computing (New York: Cambridge Univ. Press)

Reames, D. V. 1999, SSRv, 90, 413

Rouillard, A., Poirier, N., Lavarra, M., et al. 2020, ApJS, doi:10.3847/1538$4365 / \mathrm{ab} 6610$

Seripienlert, A., Ruffolo, D., Matthaeus, W. H., \& Chuychai, P. 2010, ApJ, 711,980

Servidio, S., Greco, A., Matthaeus, W. H., Osman, K. T., \& Dmitruk, P. 2011, JGRA, 116, A09102

Sreenivasan, K. R., \& Antonia, R. A. 1997, AnRFM, 29, 435

Taylor, G. I. 1938, RSPSA, 164, 476

Terasawa, T., \& Scholer, M. 1989, Sci, 244, 1050

Tessein, J. A., Matthaeus, W. H., Wan, M., et al. 2013, ApJL, 776, L8

Tessein, J. A., Ruffolo, D., Matthaeus, W. H., et al. 2015, ApJ, 812, 68

Tessein, J. A., Ruffolo, D., Matthaeus, W. H., \& Wan, M. 2016, GeoRL, 43,3620

Tooprakai, P., Seripienlert, A., Ruffolo, D., Chuychai, P., \& Matthaeus, W. H. 2016, ApJ, 831, 195

Tsurutani, B. T., \& Smith, E. J. 1979, JGRA, 84, 2773

Veltri, P., \& Mangeney, A. 1999, in AIP Conf. Proc. 471, Solar Wind Nine, ed. S. R. Habbal et al. (Melville, NY: AIP), 543

Wan, M., Matthaeus, W. H., Servidio, S., \& Oughton, S. 2013, PhPl, 20, 042307

Zank, G. P., le Roux, J. A., Webb, G. M., Dosch, A., \& Khabarova, O. 2014, ApJ, 797, 28

Zhdankin, V., Boldyrev, S., Mason, J., \& Perez, J. C. 2012, PhRvL, 108, 175004

Zhdankin, V., Uzdensky, D. A., Perez, J. C., \& Boldyrev, S. 2013, ApJ, 771,124 